\title{
Exosomal miR-665 as a novel minimally invasive biomarker for hepatocellular carcinoma diagnosis and prognosis
}

\author{
Zhen Qu ${ }^{1,3, *}$, Junhua Wu ${ }^{2, *}$, Junyi Wu ${ }^{1,2}$, Anlai Ji ${ }^{4,5}$, Guanghui Qiang ${ }^{2,5}$, Yong Jiang ${ }^{3}$, \\ Chunping Jiang ${ }^{1}$ and Yitao Ding ${ }^{1}$ \\ ${ }^{1}$ Department of Hepatobiliary Surgery, Drum Tower Hospital, Medical School of Nanjing University, Nanjing, Jiangsu Province, China \\ 2 Jiangsu Key Laboratory of Molecular Medicine, Medical School of Nanjing University, Nanjing, Jiangsu Province, China \\ ${ }^{3}$ Department of Hepatobiliary Surgery, The First People's Hospital of Changzhou, The Third Hospital Affiliated to Soochow University, \\ Changzhou, Jiangsu, China \\ ${ }^{4}$ Department of General Surgery, The Affiliated Hospital of Yangzhou University, Yangzhou, Jiangsu, China \\ ${ }^{5}$ Department of Hepatobiliary Surgery, Drum Tower Clinical College of Nanjing Medical University, Nanjing, Jiangsu Province, China \\ *These authors contributed equally to this work \\ Correspondence to: Yong Jiang, email: yjiang8888@hotmail.com \\ Chunping Jiang, email: chunpingjiang@163.com \\ Yitao Ding, email: drdingyitao0@sina.com
}

Keywords: HCC, exosomal miRNA-665, biomarker, ERK, tumor growth

Received: April 13, 2017 Accepted: August 26, $2017 \quad$ Published: September 14, 2017

Copyright: Qu et al. This is an open-access article distributed under the terms of the Creative Commons Attribution License 3.0 (CC BY 3.0), which permits unrestricted use, distribution, and reproduction in any medium, provided the original author and source are credited.

\section{ABSTRACT}

Recent studies have shown that circulating microRNAs are potential biomarkers for various types of malignancies. The aim of this study was to investigate the feasibility of using serum exosomal microRNAs (miRNAs) as novel serological biomarkers for hepatocellular carcinoma (HCC) diagnosis and prognosis. Exosomes are small membranous vesicles (30-100 $\mathrm{nm}$ ). Exosomal miR-665 levels in HCC patients were significantly higher than those in healthy subjects $(P<0.05)$, and exosomal miR-665 levels were significantly upregulated in tumours larger in size $(>5 \mathrm{~cm}$ ), in tumours with local invasion and in those at an advanced clinical stage (stage III/IV) of HCC $(P=0.0042,0.0197$, and 0.0276 , respectively). The survival time of the exosomal miR-665 high-expression group $(n=17)$ was significantly shorter than that of the low-expression group $(n=13)(P=0.036)$. In addition, we found that HCC cell-derived exosomes promoted hepatoma cell proliferation and upregulated the expression level of proteins in the MAPK/ERK pathway in vitro and in vivo. This study suggests that serum exosomal miR-665 may be a novel minimally invasive biomarker for HCC diagnosis and prognosis.

\section{INTRODUCTION}

Hepatocellular carcinoma (HCC) is the second highest cause of tumour-related death worldwide [1]. The incidence of male and female HCC cases in China is the highest in the world. Currently, a surgicalbased integrated treatment model is employed for HCC treatment, but most patients are not eligible for surgery at diagnosis. Combined with the high invasion and metastasis degree of $\mathrm{HCC}$, the prognosis of HCC patients is extremely poor $[2,3]$. Therefore, it is of great significance to identify sensitive and specific markers for the early diagnosis of HCC.
MicroRNA (miRNA) is a class of noncoding small RNA approximately $19-25$ bp long that can function at the $3^{\prime}$ non-coding region (3' untranslated region; 3'UTR) of mRNA through fully complementary or incomplete complementary means, achieving the negative regulation of genes at the post-transcriptional level by degrading mRNA or inhibiting the translation of target genes [4]. Accumulating evidence indicates that miRNAs are involved in the proliferation, metastasis and angiogenesis of tumour cells [5]. miRNAs are highly expressed in the serum of patients with many types of tumours, such as HCC [6], colorectal cancer [7], and pancreatic cancer [8], suggesting that miRNA expression in serum may be a new 
non-invasive marker for the diagnosis of multiple tumours, including HCC.

Exosomes are nanocapsule vesicles secreted by many types of cells during physiological processes and contain specific biological factors, such as miRNAs, mRNAs and proteins [9]. Exosomes can be extracted from a variety of biological fluids, including serum, urine, and ascites [10, 11]. Reports have indicated that serum miRNA is mainly contained in exosomes to avoid degradation by RNAase [12]. Therefore, exosomal miRNAs can be effective, noninvasive biological markers for disease diagnosis and prognosis determination [13-15]. However, there have been few studies exploring the value of exosomal miRNAs in the diagnosis and prognostic evaluation of $\mathrm{HCC}$ or the mechanism of specific actions of miRNA on HCC cells.

In this study, we hypothesized that exosomes from different sources contain different levels of miRNAs, which likely differentially affect HCC tumour progression. Therefore, through the evaluation of the differential expression of miRNAs in exosomes derived from hepatomas with different degrees of malignancy identified by microarray, miR-665 was identified as a miRNA with significantly differential expression in different groups. To date, there have been few reports regarding the role of miR-665 in tumours [16-18], and its role in HCC has not been reported. By detecting miR-665 expression levels in exosomes separated from the serum of HCC patients and healthy controls, we further explore the value of exosomal miR-665 in the early diagnosis and prognosis of HCC. In addition, in vitro and in vivo experiments were performed to explore the proliferative effect of exosomal miR-665 HCC cells and its potential mechanism, with the goal of facilitating the early diagnosis and prognosis prediction of HCC.

\section{RESULTS}

\section{Characterization of isolated exosomes}

To ensure the efficacy and quality of the exosomes isolated from serum and cell culture supernatants, we characterized the microvesicles by TEM, nanosight and Western blot analysis. Electron microscopic analysis of the exosomes isolated from serum and cell culture supernatant samples showed circular structures with sizes varying between 50 and $150 \mathrm{~nm}$ (Figure 1A-1E), consistent with previously reported characteristics of exosomes. Further, the identification of exosomes was confirmed by the detection of the specific exosomal protein markers CD9 and CD63 and using Western blot analysis (Figure 1F). These results confirmed the successful isolation of exosomes from different samples.

\section{Differential expression of miRNA in hepatoma cell exosomes}

First, we used miRNA microarray to screen the differentially expressed miRNAs in three exosomes isolated from cell culture supernatants of HCC samples with different degrees of malignancy. In this study, differentially expressed miRNAs were selected according to the following criteria: the fold change was no less than 1.5 for MHCC-97H exosomes vs. MHCC-97L exosomes; the fold change was no less than 5 for MHCC-97L exosomes vs. LO2 exosomes; and the $P$ value was set at 0.05 . The results suggested that $\mathrm{MHCC}-97 \mathrm{H}$ and $\mathrm{MHCC}-$ 97L exosomal miR-665 expression was higher than that in LO2 cell lines (Figure 2A). The average fold increases of miR-665 in MHCC-97H and MHCC-97L compared to the levels in the normal liver cell line were 10.1 and 5.8, respectively. RT-PCR validated the miRNA microarray results (Figure $2 \mathrm{~B}$ ). In addition, we also detected the miR-665 expression in exosomes from multiple hepatoma cell lines (HepG2, PLC and Hep3B). We found that the exosomal miR-665 expression was significantly upregulated in these three hepatoma cell lines compared with that in normal liver cells (Figure 2E).

\section{Clinical significance of exosomal miR-665 in HCC}

To understand the potential value of serum exosomal miR-665 in HCC development and progression, the expression levels of exosomal miR-665 in 30 patients with HCC and 10 healthy individuals in the follow-up cohort were determined using qRT-PCR. As expected, the expression levels of exosomal miR-665 in the HCC group were significantly increased compared with the control group (Figure 2C). Moreover, we also detected the miR665 expression in 30 pairs of HCC tissue and adjacent tissue, and the results were similar to the previous serum data. We found that miR-665 expression in HCC tissue was substantially higher than that in adjacent tissue and normal liver tissue (Figure 2D).

To better understand the potential roles of serum exosomal miR-665 in HCC development and progression, we validated the clinicopathological significance of miR-665 expression in serum exosomes by RT-PCR. We categorized $30 \mathrm{HCC}$ cases into two groups according to the mean miR-665 expression level in preoperative serum exosomes. In this study, a 5-fold increase was used as the cut-off value to divide the samples into high-miR665 -expression ( $\geq 5$-fold) and low-miR-665-expression $(<5$-fold) groups. The statistical analysis revealed that, compared with the low-miR-665-expression group, the high-expression group showed higher clinical stages $(P=0.0276)$ and stronger tendencies of association with larger tumour size ( $>5 \mathrm{~cm} ; P=0.0042)$ and local tumour invasion and metastases $(P=0.0197$, Table 1). However, there was no correlation of exosomal miR-665 expression with other clinical features, including age, sex, HBV infection and AFP level $(P>0.05)$. Furthermore, we examined the association between serum exosomal miR-665 expression and HCC patient prognosis using 30 primary HCCs with hepatectomy. Patients with exosomal miR-665 expression levels less than the 5-fold 
were assigned to a low-expression group $(n=13)$ and those above 5-fold to a high-expression group $(n=17)$. Further investigation demonstrated that the high-miR-665expression group had a significantly poorer prognosis than the low-expression group $(P<0.05$, Figure 3$)$.

\section{Exosomal miR-665 promotes HCC cell proliferation}

Because high exosomal miR-665 expression was associated with the malignant potential of HCC, the function of exosomal miR-665 was assessed using an MTT assay. The MTT results showed that HCC-derived exosomes promoted the proliferation of SMMC-7721 cells in a time-dependent manner, and exosomes derived from tumour cells with higher exosomal miR-665 levels had

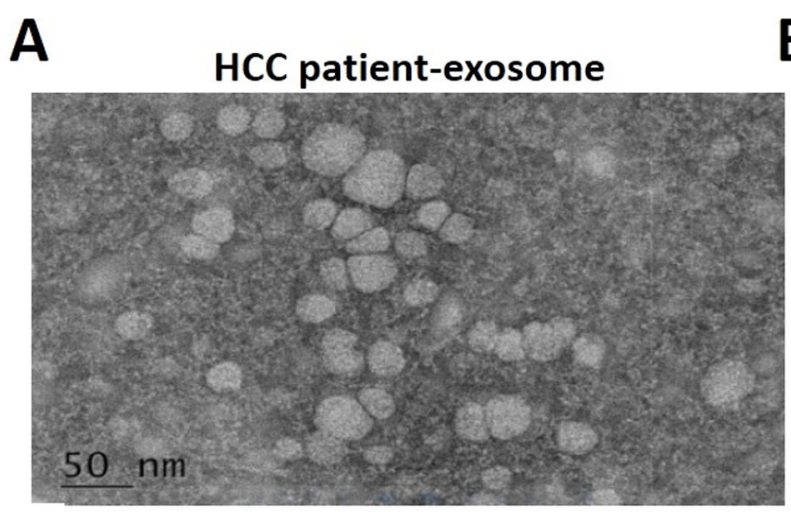

greater efficacy. After the application of anti-miR-665, the cell proliferation-promoting effects of exosomes were suppressed $(P<0.05$, Figure 4$)$.

\section{Exosomal miR-665 affects cell proliferation and tumour growth through the MAPK/ERK pathway}

To investigate whether the effect of exosomal miR665 on HCC cell proliferation was related to the activation of ERK and other proteins, we detected the expression levels of p-ERK, p-STAT3, and EGFR in SMMC-7721 cells using Western blotting. The results showed that exosomes could upregulate the expression of p-ERK, and MHCC97H-derived exosomes caused the most significant effects (Figure 5A). In addition, HCC-derived exosomes

B

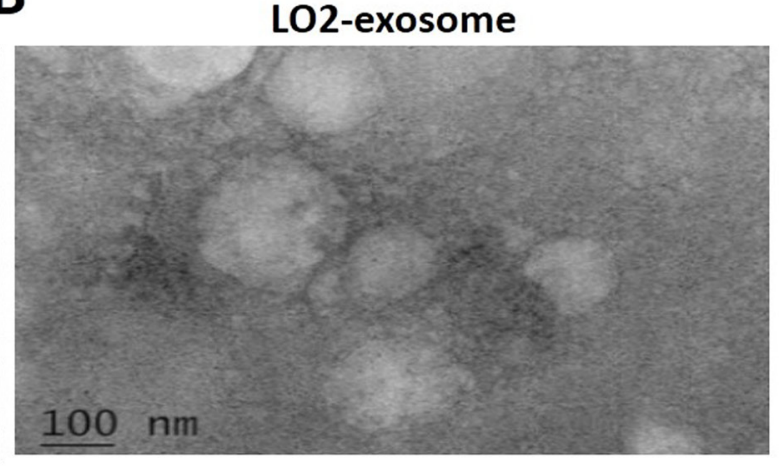

C

D

MHCC-97H-exosome

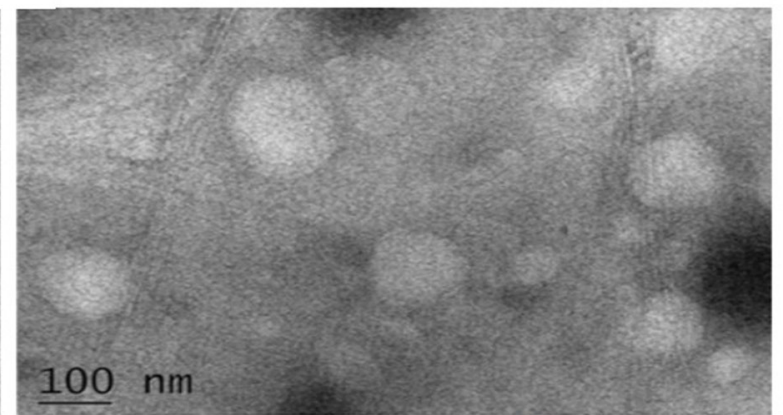

$\underline{100} \mathrm{~nm}$

E
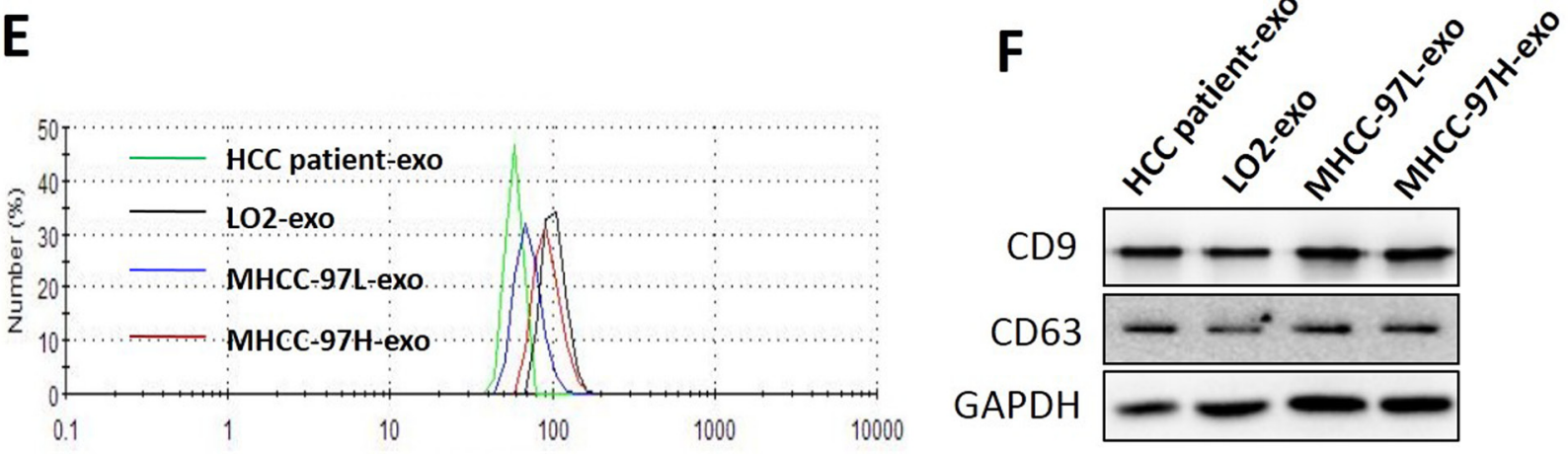

Figure 1: Characterization of isolated exosomes. Exosomes were purified from the serum of HCC patients (A), LO2 (B), MHCC97L (C) and MHCC-97H (D) cell culture supernatant and examined by TEM. (E) Size distribution analysis of purified exosomes using a Nanosight instrument. (F) Exosome markers (CD9, CD63) were analysed using Western blot. The data demonstrated that extracts were enriched with exosomal marker proteins CD9 and CD63. 
Table 1: Correlations of patient clinicopathologic characteristics with exosomal miR-665 expression level

\begin{tabular}{|c|c|c|c|c|c|}
\hline \multirow{2}{*}{\multicolumn{3}{|c|}{ Clinicopathological features }} & \multicolumn{2}{|c|}{ Exosomal miR-665 } & \multirow[t]{3}{*}{$P$ value } \\
\hline & & & \multirow{2}{*}{$\frac{\text { Low } \boldsymbol{n}(\mathbf{\%})}{8(44.4)}$} & \multirow{2}{*}{$\frac{\text { High } \boldsymbol{n} \mathbf{( \% )}}{10(55.6)}$} & \\
\hline Sex & $\mathrm{F}$ & 18 & & & \\
\hline & M & 12 & $5(41.7)$ & $7(58.3)$ & $P=0.8804$ \\
\hline \multirow[t]{2}{*}{ Age } & $>60$ & 12 & $5(41.7)$ & $7(58.3)$ & \\
\hline & $\leq 60$ & 18 & $8(44.4)$ & $10(55.6)$ & $P=0.8804$ \\
\hline \multirow[t]{2}{*}{ HBsAg } & + & 24 & $11(45.8)$ & $13(54.2)$ & \\
\hline & - & 6 & $2(33.3)$ & $4(66.7)$ & $P=0.5805$ \\
\hline \multirow[t]{2}{*}{$\operatorname{AFP}(\mathrm{ng} / \mathrm{mL})$} & $>20$ & 22 & $10(45.5)$ & $12(54.5)$ & \\
\hline & $\leq 20$ & 8 & $3(37.5)$ & $5(62.5)$ & $P=0.6974$ \\
\hline \multirow[t]{2}{*}{ Cirrhosis } & + & 20 & $8(40.0)$ & $12(60.0)$ & \\
\hline & - & 10 & $5(50.0)$ & $5(50.0)$ & $P=0.6023$ \\
\hline \multirow[t]{2}{*}{ Tumour size $(\mathrm{cm})$} & $>5$ & 20 & $5(25.0)$ & $15(75.0)$ & \\
\hline & $\leq 5$ & 10 & $8(80.0)$ & $2(20.0)$ & $P=0.0042$ \\
\hline \multirow[t]{2}{*}{ Capsule invasion } & + & 9 & $1(11.1)$ & $8(88.9)$ & \\
\hline & - & 21 & $12(57.1)$ & $9(42.9)$ & $P=0.0197$ \\
\hline \multirow[t]{2}{*}{ TNM stage } & III-IV & 24 & $8(33.3)$ & $16(66.7)$ & \\
\hline & I-II & 6 & $5(83.3)$ & $1(16.7)$ & $P=0.0276$ \\
\hline
\end{tabular}
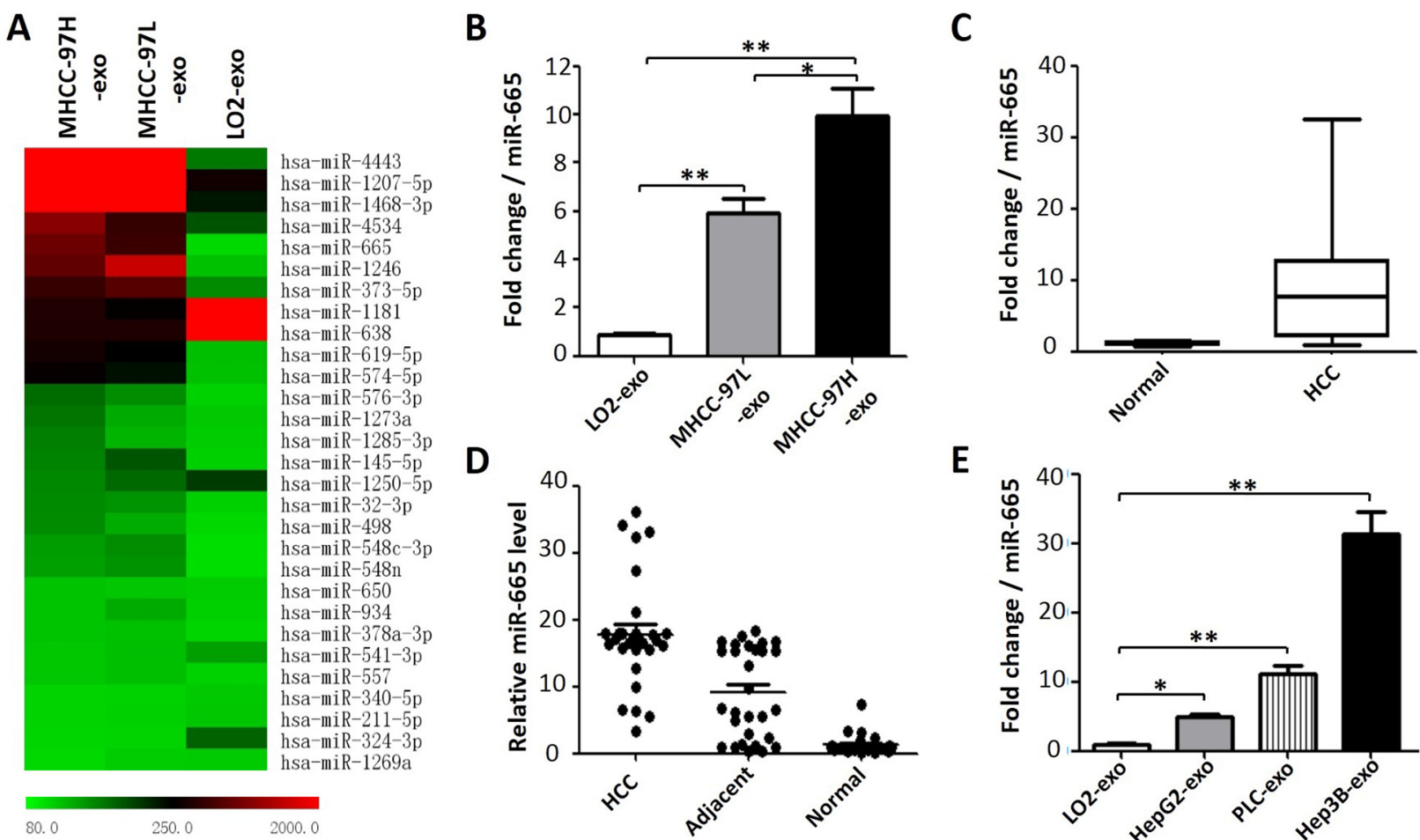

$\mathbf{E}$

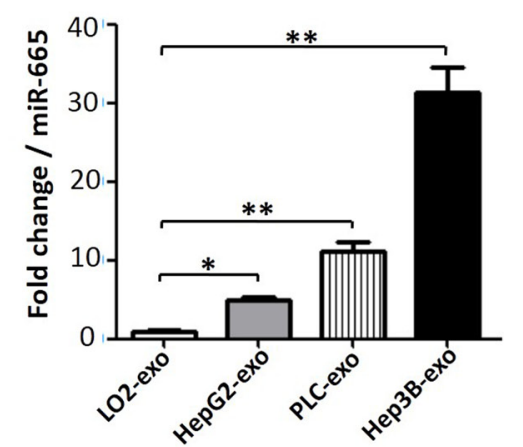

Figure 2: Exosomal miR-665 expression is significantly higher in HCC. (A) A heatmap representing the differential miRNA expression in exosomes derived from three cell lines. The colour "red" indicates high relative expression, and "green" indicates low relative expression. (B) RT-PCR validated the reliability of the miRNA microarray, the average fold increase of exosomal miR-665 in MHCC-97H and MHCC-97L cells vs. the normal liver cell line (LO2) were 10.1 and 5.8, respectively. $* P<0.05$; $* * P<0.01$. (C) The levels of exosomal miR-665 in serum samples from 30 patients with HCC and 10 healthy controls were determined by RT-PCR. (D) miR-665 expression in HCC, adjacent and normal liver tissue. The absolute $2^{-\Delta \Lambda C T}$ values are presented. (E) miR-665 expression was detected by RT-PCR in the exosomes derived from multiple hepatoma cell lines (HepG2, PLC and Hep3B). 


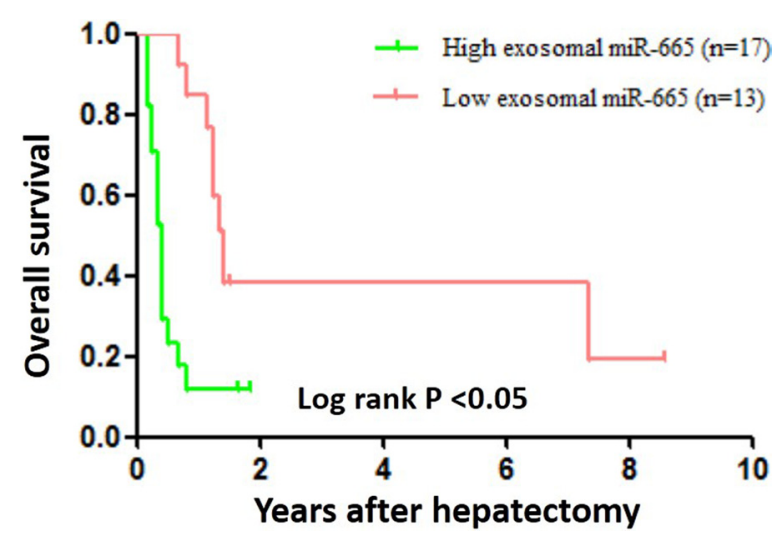

Figure 3: Overall survival curves for 30 HCC patients who underwent hepatectomy. The patients with high exosomal miR-665 expression exhibited significantly poorer long-term prognosis after hepatectomy.
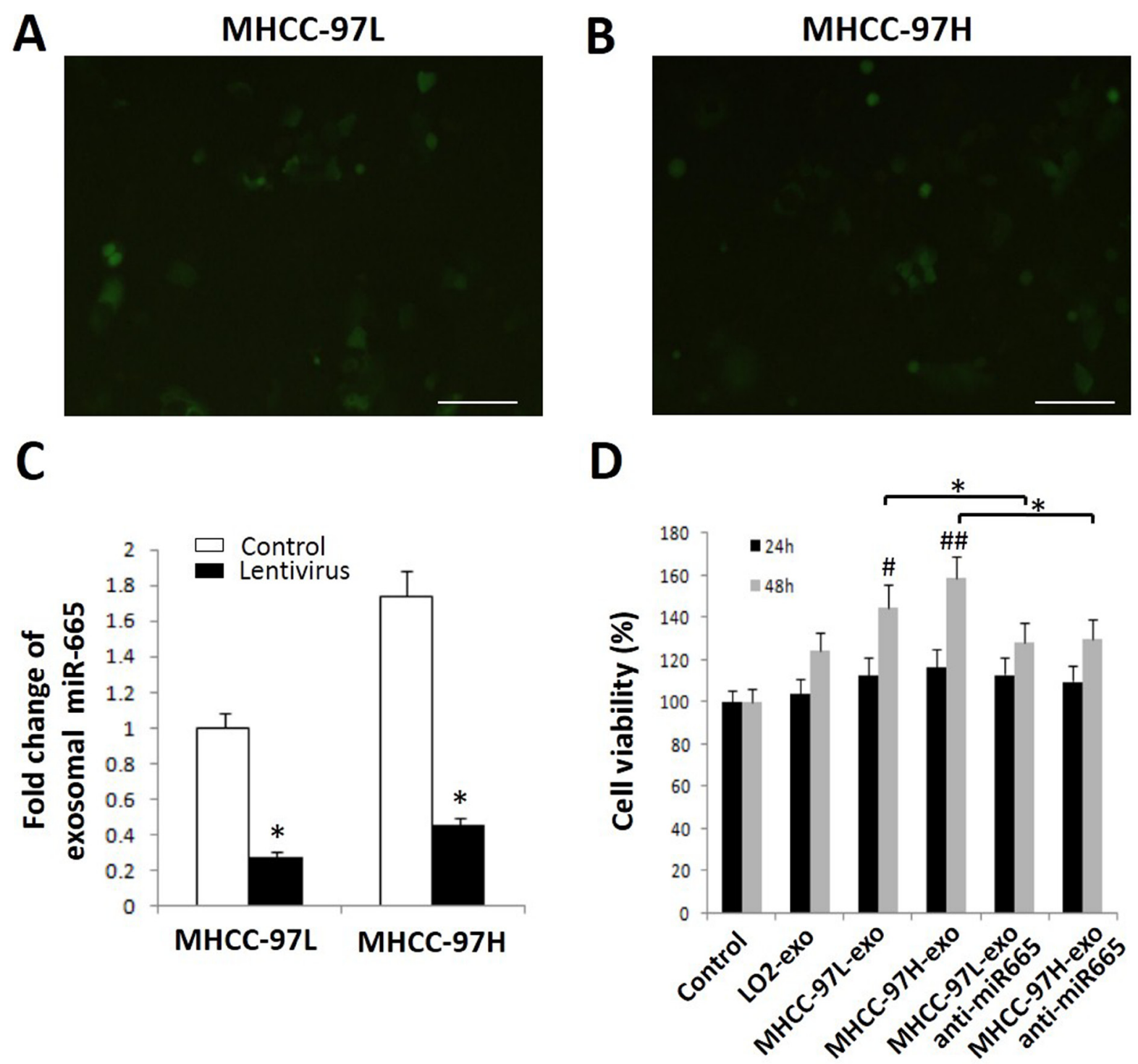

Figure 4: Exosomal miR-665 promotes cell proliferation in HCC cells. Micrographs of MHCC-97L (A) and MHCC-97H cells (B) infected with lentivirus-green fluorescent protein, captured after $72 \mathrm{~h}$ under a fluorescence microscope. Scale bar represents $500 \mu \mathrm{m}$. (C) miR-665 expression in the lentivirus group and control group. ${ }^{*} P<0.05$. (D) Cell viability was assessed using an MTT assay. HCC exosomes promote cell proliferation in HCC cells; after the application of anti-miR-665, the cell proliferation-promoting effects of exosomes were downregulated. Equal volume of PBS was used as a control. $* P<0.05$. 
contained many different molecules (Figure 5B). These results suggested that HCC-derived exosomes may also transmit these factors to influence the microenvironment.

To detect whether exosomal miR-665 could promote tumour growth in liver cancer in vivo, we established a subcutaneous xenograft model in nude mice and injected MHCC-97H-derived exosomes or MHCC-97H exosomeanti-miR-665 into mouse subcutaneous tumours. As shown in Figure 6, the tumours in mice treated with MHCC-97H-derived exosomes were significantly larger than control groups; this effect was obviously weakened in the exosome-anti-miR-665 group, indicating that exosomal miR-665 could promote HCC growth and progression. Figure $6 \mathrm{E}$ shows the tumour volume of each group; it is clear that the tumour volume in mice treated with MHCC-97H-exosomes was significantly greater than that in other groups. Furthermore, we detected the expression of p-ERK in mouse subcutaneous tumours using immunohistochemistry. The results showed that
MHCC-97H-exosomes upregulated the expression of p-ERK, and the knock-down of exosomal miR-665 expression obviously weakened this effect (Figure 7).

\section{DISCUSSION}

Currently, the clinical diagnosis of HCC depends on AFP and imaging examination, such as colour Doppler ultrasound, computed tomography (CT), magnetic resonance imaging (MRI), and tumour cytological biopsy. However, an early diagnosis of HCC is still difficult [19], and many patients are not eligible for surgery at diagnosis. Therefore, the identification of new and sensitive biomarkers for the early diagnosis of HCC is urgently needed.

Many studies have confirmed that miRNAs are involved in tumour formation and development [20, 21]. Studies have suggested that serum miR-185 can not only be used as a marker for early-stage $\mathrm{HCC}$ but is also
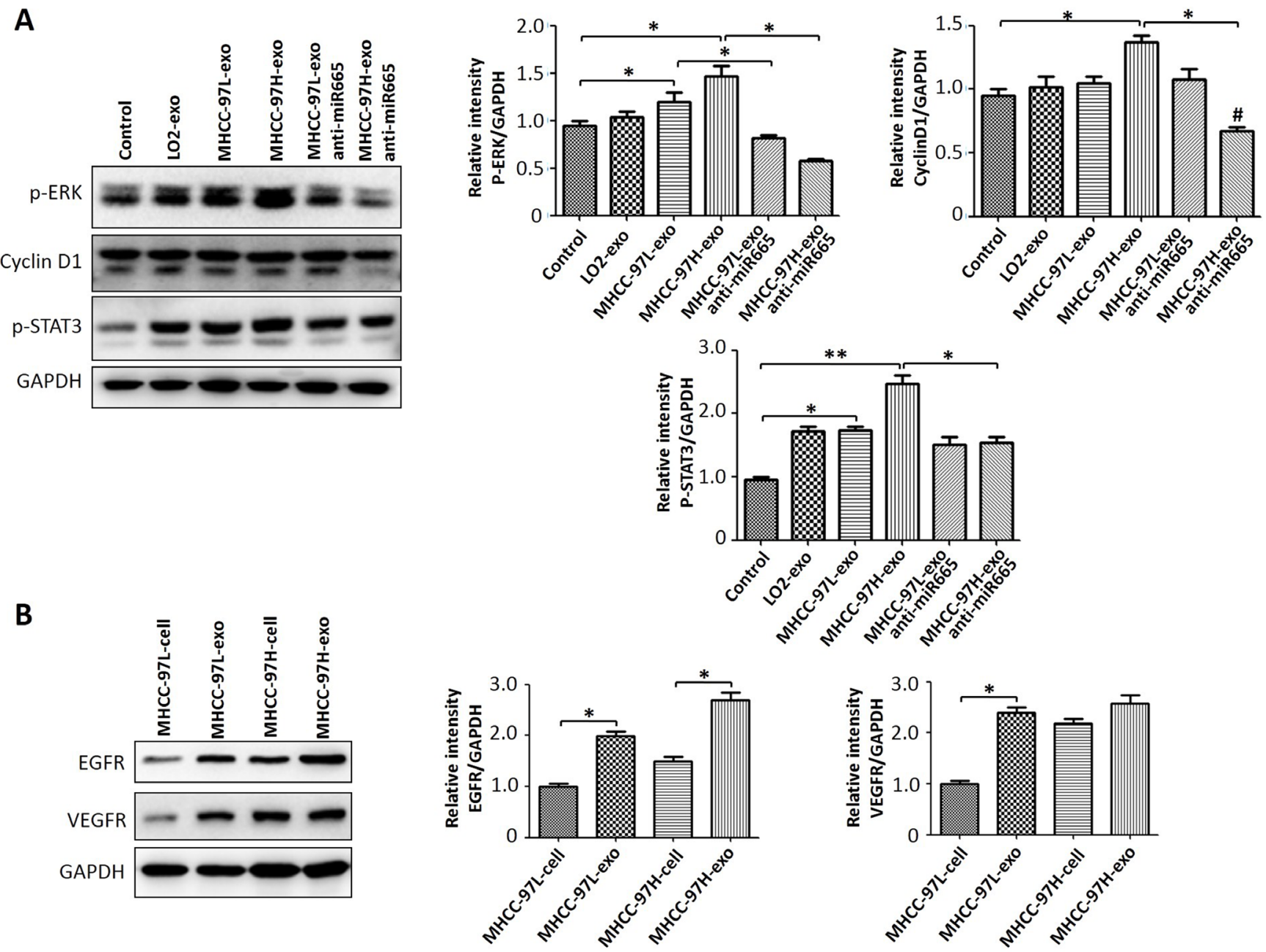

Figure 5: Exosomal miR-665 upregulated the expression of HCC cell proliferation-related proteins. (A) The expression levels of p-ERK, CyclinD1 and p-STAT3 were detected by Western blot; an equal volume of PBS was used as a control. Exosomal miR-665 could promote HCC cell proliferation through activating the MAPK/ERK pathway; the effects of exosomes decreased with the application of anti-miR-665 in HCC cells. (B) The expression levels of VEGFR and EGFR were detected by Western blot. HCC-derived exosomes contained many different molecules (VEGFR and EGFR). $* P<0.05$. 
closely related to liver metastasis and tumour size [22]. However, the molecular mechanism and function of most HCC-related miRNAs remain unclear. Exosomes derived from different tumours contain miRNA with specific characteristics. Recipient cells demonstrate obvious tumourigenic tendencies after cellular uptake of such miRNAs, including tumour cell migration, immune response, angiogenesis, and the infiltration and metastasis of tumour cells $[23,24]$. A previous study found that the levels of miR-21 and miR-146a in exosomes derived from cervical cancer were significantly elevated [25]. Liu et al utilized diethylnitrosamine to induce the establishment of a rat HCC model and found that miRNAs of the combined cycle (miRNA-10b, miRNA-21, miRNA-122, and miRNA-200a) and exosomes can be used to effectively diagnose early-stage HCC [26]. Xiang et al found that tumour-derived exosomal miR-222-3p is an effective regulator in the polarization of tumours and may be a biomarker of epithelial ovarian cancer [27]. Another study showed that the serum exosomal miRNAs of patients might be used as effective biomarkers in predicting $\mathrm{HCC}$ recurrence through the detection of serum exosomal miRNA levels in liver transplantation patients. It was found that exosomal miR-718 level is associated with tumour recurrence, metastasis and prognosis [28]. In summary, understanding the functions of miRNAs with abnormal expression in $\mathrm{HCC}$ and their related mechanisms, especially the identification of miRNAs with abnormal expression, by high-throughput sequencing screening is particularly important, and this information may improve our understanding of the development mechanisms of $\mathrm{HCC}$ and provide new targets for $\mathrm{HCC}$ treatment.

Previous studies are often limited to in vitro or animal experiments, while the mechanism of action and clinical evidence for the role of exosomes and miRNA remain unclear. The current study utilized a miRNA microarray chip to identify exosomal miRNAs with significantly different expression levels in different hepatoma cell lines and found that exosomal miR-665 derived from different cells showed significantly different expression levels. qRT-PCR validation found that the exosomal miR-665 levels in MHCC-97H cells with a high degree of malignancy were 2 - and 10-fold those in

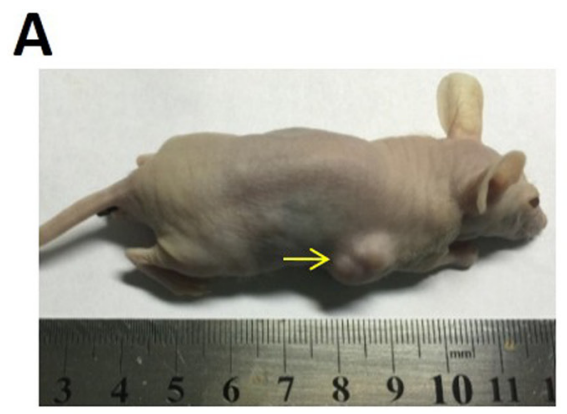

Control

D

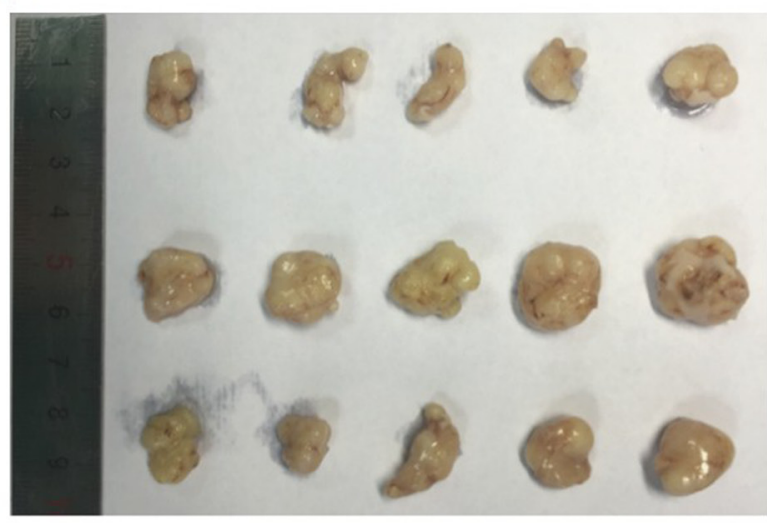

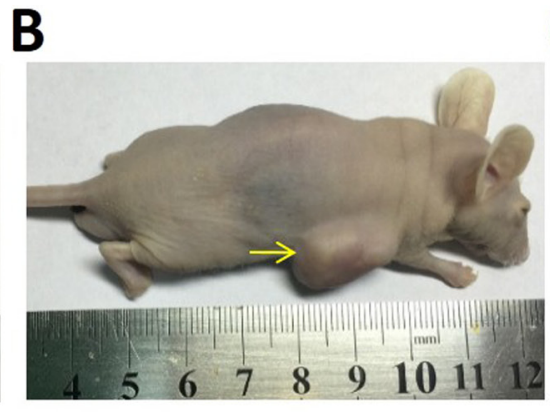

MHCC-97H-exo

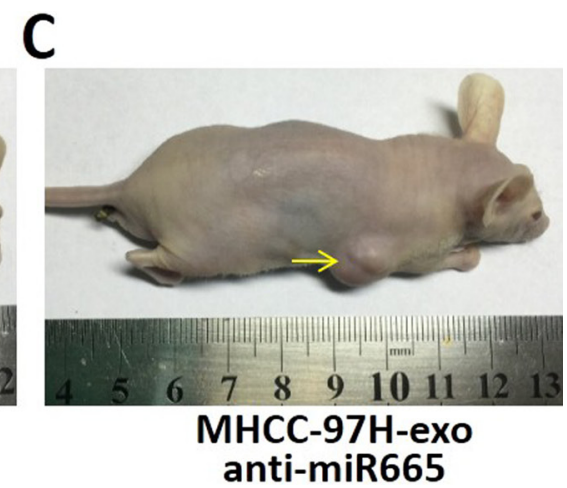

$\mathbf{E}$
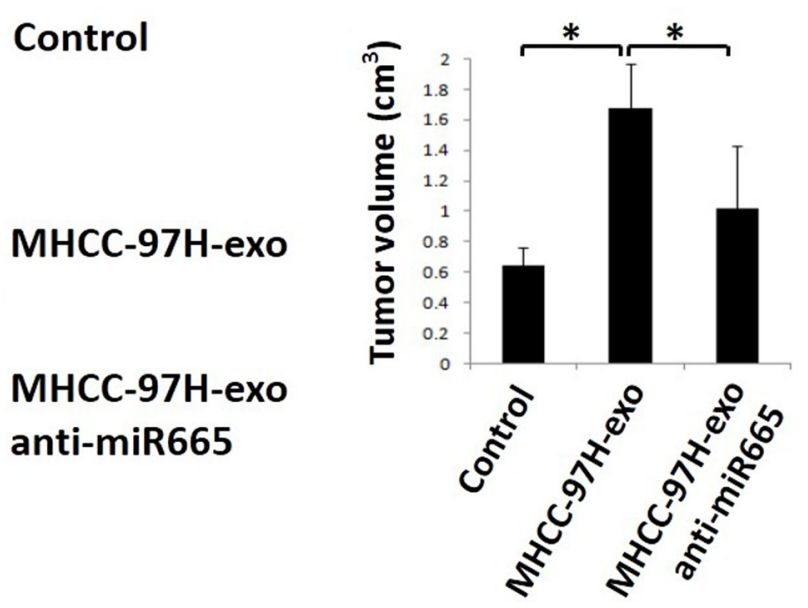

Figure 6: Exosomal miR-665 promoted xenograft tumour growth in vivo. The size of tumours at the end of the experiment from mice treated with PBS (Control, A), MHCC97H-exosome (B) and MHCC97H-exosome anti-miR-665 (C, D, E) Representative images and tumour volume changes in the mice bearing MHCC97H-exosome or MHCC97H- exosome anti-miR-665. $n=5$, significant difference between groups are shown as $* P<0.05$. 
MHCC-97L and LO2 cells, respectively. In addition, qRT-PCR was used to detect serum exosomal miR-665 levels in clinical HCC patients and normal controls and found that the exosomal miR-665 levels in the serum of HCC patients was significantly higher than that of the healthy control group. Furthermore, the exosomal miR665 level closely correlated with the clinicopathological parameters of patients, such as clinical stage, tumour differentiation, and patient survival, suggesting that serum exosomal miR-665 may be extensively involved in HCC occurrence and development. In addition, previous studies have found that exosomes may transmit VEGF and miRNAs and promote tumour progression and metastasis (by affecting cell phenotype changes, etc.), suggesting that exosomes and key miRNAs are closely involved
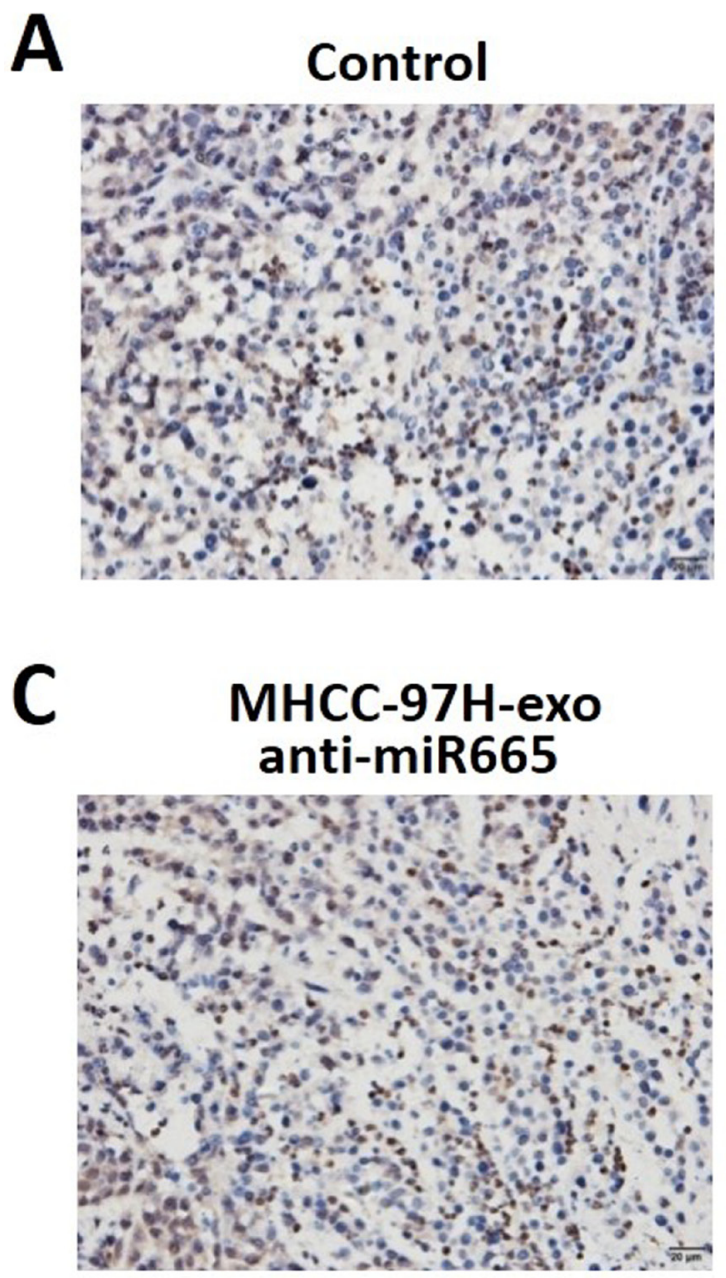

in tumour progression and may be a potential tumour therapeutic target $[29,30]$.

Skog et al [31] reported that exosomes released by primary cultured malignant glioma cells could promote the proliferation of human glioma cells. An in vivo study also confirmed that exosomes derived from mouse breast cancer cells could inhibit the function of NK cells and promote tumour growth [32]. The research of Takayuki et al showed that exosome-mediated miRNA transport play an important role in the communication process among HCC cells, promoting tumour growth and metastasis, the mechanism of which may be related to regulation of transforming growth factor B activated kinase-1 (TAK1) expression by exosomes derived from HCC cells and the related signalling pathways [24]. These results suggest that

B

\section{MHCC-97H-exo}

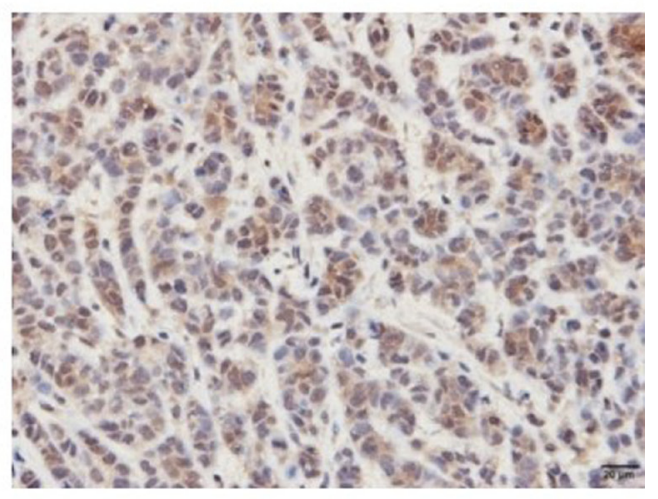

D

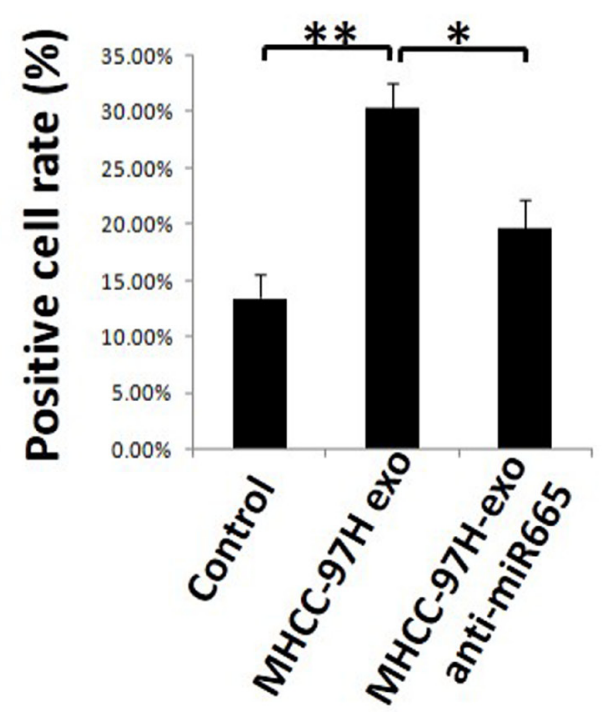

Figure 7: MHCC97H-exosome anti miR-665 downregulated the expression of P-ERK in vivo. Tumours from mice treated with PBS (Control, A), MHCC97H-exosome (B) and MHCC97H-exosome anti-miR-665 (C) were paraffin-embedded and sectioned, followed by staining by using immunohistochemistry method. (D) The number of P-ERK-positive cells notably decreased in the MHCC97Hexosome anti-miR-665 group compared with the number in the MHCC97H-exosome group. $* P<0.05$. 
tumour-derived exosomes play an important role in the process of tumour cell proliferation and metastasis. Our study found that exosomes derived from different HCC cell lines could promote the proliferation of the HCC cell line SMMC-7721 in a co-culture. The MAPK/ERK signal pathway is an important intracellular proliferative and anti-apoptotic pathway and plays an important role in the malignant proliferation of tumour cells by affecting the activity of effector molecules, such as downstream cell cycle regulatory proteins and apoptosis-related proteins $[33,34]$. Recent studies have indicated that MAPK/ERK signalling molecules are overexpressed or consistently activated in gastric cancer [35], colon cancer, breast cancer [36, 37], and other tumours. Our results showed that the exosomes of HCC cells activated the MAPK/ERK pathway when promoting the proliferation of SMMC7721 cells, and the expression of proteins in the MAPK/ ERK pathway was reduced when anti-miR-665 was introduced, suggesting that tumour-derived exosomes may activate the MAPK/ERK pathway through miR-665 and further promote the proliferation of tumour cells. In addition, the analysis of exosomal protein showed that it contained VEGF, which was closely related to the malignant proliferation of tumour cells. By increasing the expression of growth factor surface receptors through binding with tumour cell surface receptors or directly fusing with the cell membrane, the expression of these molecules is likely to play a role in promoting proliferation.

Although the physiological role of tumour-cellderived exosomal miR-665 remains unclear, it is certain that exosomal miR-665 plays an important role in the in vivo growth and development of tumour cells. The results of the current study showed that tumouroriginated exosomal miR-665 could promote tumour cell proliferation in vitro, and the mechanism may be related to activation of the MAPK/ERK pathway by biological factors inside the exosomes, such as VEGF. Because exosomes are bioactive structures actively produced and secreted by living cells, they contain a variety of biological factors, including mRNAs and miRNAs, and demonstrate complicated and changeable functions [38]. Therefore, an in-depth understanding of the molecular mechanisms of tumour-derived exosomes in promoting HCC cell proliferation has important significance for clarifying of the role of exosomes in the occurrence and development of $\mathrm{HCC}$ and their potential therapeutic value.

\section{MATERIALS AND METHODS}

\section{Cells and reagents}

MHCC-97H, MHCC-97L and LO2 cell lines were obtained from the Liver Cancer Institute, Fudan University (Shanghai, China). SMMC-7721 cell line was purchased from Cell Bank of Xiangya Central Experiment Laboratory of Central South University (Changsha, China). MHCC97H, MHCC-97L and SMMC-7721 were maintained in Dulbecco's Modified Eagle Medium (DMEM, WISENT, CA, USA) containing 10\% foetal bovine serum (FBS) (ExCell Bio, China). LO2 cell line was maintained in 1640 containing 10\% foetal bovine serum (FBS) (ExCell Bio, China). All cells were incubated in $5 \% \mathrm{CO}_{2}$ at $37^{\circ} \mathrm{C}$. MTT (3-(4,5-dimethylthiazol) 2, 5-diphenyltetrazolium) was purchased from Sigma Aldrich (St. Louis, MO, USA). The antibodies for glyceraldehyde-3-phosphate dehydrogenase (GAPDH), p-Akt, Akt, p-ERK, p-JNK, p-P38 and EGFR were obtained from Cell Signaling Technology (Beverly, MA, USA). The antibody for VEGFR-2 was obtained from Bioworld Technology Inc. (Bioworld, USA).

\section{Patient samples}

The thirty serum specimens and HCC tissue specimens were collected from $\mathrm{HCC}$ patients in the Department of Hepatobiliary Surgery of the Affiliated Drum Tower Hospital, Medicine School of Nanjing University (Nanjing, China). No patients received any anti-cancer treatment or had any other endocrine, immune, or metabolic diseases. The tumour type and the grade of cell differentiation were diagnosed based on the criteria of the World Health Organization (WHO), whereas the pathological stage of each tumour was determined by the International Union Against Cancer (UICC) TNM classification. The normal control group contained ten samples from healthy people. Written informed consent was obtained from all subjects prior to the recruitment. The study protocol was approved by the Institutional Review Board of the hospital ethics committee. The clinical characteristics of the subjects are listed in Table 2.

\section{Isolation of exosomes}

Peripheral blood was collected and centrifuged at $3,000 \mathrm{rpm}$ for $10 \mathrm{~min}$ at $4^{\circ} \mathrm{C}$ to spin down the blood cells. The supernatants were centrifuged at $12,000 \mathrm{~g}$ for $10 \mathrm{~min}$ at $4^{\circ} \mathrm{C}$ to completely remove the cellular components. The serum samples were stored at $-80^{\circ} \mathrm{C}$ until use. Exosomes were isolated from serum samples using Exoquick TM Kit (System Biosciences, USA) following the manufacturer's protocol. Briefly, $0.1 \mathrm{~mL}$ of Total Exoquick TM Reagent was added to $0.4 \mathrm{~mL}$ of serum and incubated for $12 \mathrm{~h}$ at $4^{\circ} \mathrm{C}$, followed by centrifugation at $10,000 \times \mathrm{g}$ for $30 \mathrm{~min}$ at room temperature. Finally, the exosome pellets were collected for characterizations and RNA extractions.

Different cell lines were cultured in media with 10\% exosome-free FBS (by ultracentrifugation overnight). After $48 \mathrm{~h}$, cell culture media were collected, and exosomes were isolated from the supernatant by differential centrifugation as previously described (see "Isolation of exosomes") [39]. 
Table 2: Clinical characteristics of the subjects $(n=40)$

\begin{tabular}{lccc}
\hline Variables: median $($ range $)$ & Healthy control $(\boldsymbol{n}=\mathbf{1 0})$ & HCC $(\boldsymbol{n}=\mathbf{3 0})$ & $\boldsymbol{P}$-value \\
\hline Sex $(\mathrm{M} / \mathrm{F})$ & $6 / 4$ & $18 / 12$ & $P=1.0$ \\
Age (years) & $53.0 \pm 2.2$ & $54.3 \pm 2.4$ & $P=0.36$ \\
ALT & 27.4 & 38.5 & $P=0.031$ \\
AST & 25.7 & 40.2 & $P=0.026$ \\
AFP (ng/ml) & 2.8 & 1083.8 & $P<0.001$ \\
HBsAg-positive & 2 & 24 & $P<0.001$ \\
Tumour size (cm) & & 4.5 & \\
Tumour number $(1 / 2 / 3 \sim)$ & & $21 / 5 / 4$ & \\
Capsule invasion (+/-) & & $9 / 21$ & \\
TNM stage (I-II/III-IV) & & $6 / 24$ & \\
\hline
\end{tabular}

\section{Transmission electron microscopy}

The extracted pellets were observed under transmission electron microscopy (TEM) as previously described. A drop of purified exosomes (approximately $10 \mu \mathrm{L}$ ) was fixed with $1 \%$ glutaraldehyde for $10 \mathrm{~min}$, washed, and contrasted in $2 \%$ uranyl acetate. Images were obtained by TEM (JEM-2100, Jeol, Japan), and the size distribution of the isolated exosomes was analysed by Nanosight instrument (Malvern, UK) according to the manufacturer's instructions.

\section{Western blot analysis}

To determine the level of indicated proteins, exosomes and hepatoma cells were lysed with RIPA peptide lysis buffer (Beyotime Biotechnology, China) containing $1 \%$ protease inhibitors (Pierce). Twenty micrograms of proteins was analysed by Western blot as described. The PVDF membranes with transferred proteins were incubated with primary antibodies at $4^{\circ} \mathrm{C}$ overnight and HRP-conjugated secondary antibodies at room temperature for $2 \mathrm{~h}$. The signal was developed by the enhanced chemiluminescence (ECL) reagent (Millipore, Bedford, MA, USA) and visualized by FluorChem FC2 Imaging System (Alpha Innotech, San Leandro, CA, USA).

\section{miRNA microarray analysis}

For the miRNA expression analyses, we used the miRNA microarray to process the samples (Super Biotek, China). A TaqMan Low Density Array v3.0 (Applied Biosystems) was used to detect and quantify up to 291 human miRNAs using an Applied Biosystems real-time instrument, according to the manufacturer's protocol, and the data were analysed further with Agilent GeneSpring GX 10 Microarray Data Analysis Software. In this study, differentially expressed miRNAs were selected according to the following criteria: the change fold was no less than 1.5-times for MHCC-97H exosomes vs. MHCC-97L exosomes and 5-times for MHCC-97L exosome vs. LO2 exosome; the $P$ value was set at 0.05 .

\section{Quantitative RT-PCR}

Total RNA was isolated from the exosomal pellets. To validate miRNA expression, real-time quantitative PCR (qRT-PCR) was performed using SYBR Premix Dimer-Eraser kit (Takara Biotechnology, Dalian, China) on an ABI Prism 7300 HT Detection System (Applied Biosystems, CA, USA). U6 snRNA was used as an internal control. The primers for miR-665 and U6 were purchased from RiboBio (Guangzhou, China). The primers used are listed as follows: miR-665 (5'-GTCGTATCCAGTG CAGGGTCCGAGGTATTCGCAC TGGATACGACAG GGGC-3'), U6 (5'-GTCGTATCCAGTGCAGGGTCCG AGGTATTCGCACTGGATACGACAAAATA-3'). The relative gene expression values for the target miRNA were normalized to U6 snRNA and calculated using the $2^{-\Delta \Delta \mathrm{CT}}$ method.

\section{Lentivirus vector transfection}

To obtain hepatoma cell-derived exosomes with downregulated miR-665, HCC cell lines (MHCC-97L and MHCC-97H) were seeded into a 6-well plate at a density of $5 \times 10^{5}$ cells/well to a final volume of the culture solution of $2 \mathrm{~mL}$. Cells were transfected at a multiplicity of infection (MOI) of 10 , incubated at $37^{\circ} \mathrm{C}$ for $5 \mathrm{~h}$ and allowed to recover in fresh culture medium for $72 \mathrm{~h}$ at $37^{\circ} \mathrm{C}$ with $5 \% \mathrm{CO}_{2}$, under the excitation wavelength $\mathrm{HCC}$ cells displayed green colour with green fluorescence microscopy.

\section{Cell viability assay}

Cell viability was monitored using MTT assay. Generally, $5 \times 10^{3}$ cells were allowed to grow in 96-well plates. After incubation with tumour-derived exosomes for 24 and $48 \mathrm{~h}, 20 \mu \mathrm{L}$ of MTT solution $(0.5 \%)$ was added 
to the medium for further incubation for $4 \mathrm{~h}$. A total of $150 \mu \mathrm{L}$ of DMSO was added to every well to dissolve the insoluble formazan product after removing the medium. The absorbance of the coloured solution was measured at $570 \mathrm{~nm}$ with a spectrophotometer. All experiments were performed in triplicate.

\section{Animal model}

All animal procedures were performed according to national guidelines and approved by the Animal Care Ethics Committee of Nanjing Drum Tower Hospital. Fifteen male BALB/c nu/nu mice (4-6 weeks old, Laboratory Animal Center of Shanghai, Academy of Science). All mice received subcutaneous injections of SMMC-7721 cells in the right armpit $\left(1 \times 10^{7}\right.$ cells in $200 \mu \mathrm{L}$ of PBS per mouse $)$. Fifteen mice were randomly divided into three groups (the control group, MHCC-97H-exosome group, and the MHCC-97H-exosome anti-miR665 group) when the tumours reached a volume of $50-100 \mathrm{~mm}^{3}$ (15 days after subcutaneous injections of tumour cells), subcutaneously injected with exosomes $(100 \mu \mathrm{g}$ of total protein, in the vicinity of the subcutaneous tumours), respectively, the control group was injected with equal volume PBS. The mice were examined every 2 days, and all mice were sacrificed by cervical dislocation under general anaesthesia with chloral hydrate $(5 \%, 100 \mu \mathrm{L} / 10 \mathrm{~g})$.

\section{Immunohistochemical}

Formalin-fixed and paraffin-embedded subcutaneous tumour samples from nude mice were first cut into 5- $\mu \mathrm{m}-$ thick sections. Then, antigen retrieval was accomplished by deparaffinization, rehydration, and boiling in a microwave oven with citrated buffer. Hydrogen peroxide $(3 \%)$ in PBS was used to block endogenous peroxidase activity, and BSA was used to block nonspecific staining. Sections were incubated with an anti-p-ERK polyclonal antibody (1: 200, bs-3292R, Bioss, Beijing, China) at $4^{\circ} \mathrm{C}$ overnight. The EliVision plus Kit (kit-9902, Maixin Biotech, China) was used to detect primary antibody followed by staining with DAB reagent and counterstaining with haematoxylin. Finally, the slides were imaged under the microscope (BX43, OLYMPUS, Japan).

\section{Statistics}

All results, except the microRNA value, are described as the mean \pm s.d. The relationships among miR665 expression, clinicopathological factors and the in vitro assay data were analysed using Student's $t$-tests, $\mathrm{X}^{2}$-tests and ANOVA. Post-operative survival curves were plotted using the log-rank test. All differences were considered significant at the level of $P<0.05$. All statistical analyses were performed using SPSS (Statistical Package for the Social Sciences) for Windows release 18.0 (SPSS Inc., Chicago, IL, USA).

\section{ACKNOWLEDGMENTS AND FUNDING}

We thank the Translational Medicine Core facilities of the Medical School of Nanjing University for instrument support. This study was supported by the National Natural Science Foundation of China (no. 81300337, 81572393, $81472820,81172143,11334004$, and 81421091), the National Basic Research Program of China (973 Program, 2013CB834100), the Key Project supported by Medical Science and Technology Development Foundation, Nanjing Municipality Health Bureau (no. ZKX15020 and ZKX 12011), the Natural Science Foundation of Jiangsu Province (no. BK20141324), Jiangsu special program for clinical medical science and technology (BL2014054).

\section{CONFLICTS OF INTEREST}

There are no conflicts of interest to declare.

\section{REFERENCES}

1. Jemal A, Bray F, Center MM, Ferlay J, Ward E, Forman D. Global cancer statistics. CA Cancer J Clin. 2011; 61:69-90.

2. El-Serag HB, Rudolph KL. Hepatocellular carcinoma: epidemiology and molecular carcinogenesis. Gastroenterology. 2007; 132:2557-2576.

3. El-Serag HB: Hepatocellular carcinoma. N Engl J Med. 2011; 365:1118-1127.

4. Lewis BP, Burge CB, Bartel DP. Conserved seed pairing, often flanked by adenosines, indicates that thousands of human genes are microRNA targets. Cell. 2005; 120:15-20.

5. Ruan K, Fang X, Ouyang G: MicroRNAs: novel regulators in the hallmarks of human cancer. Cancer Lett. 2009; 285:116-126.

6. Qi J, Wang J, Katayama H, Sen S, Liu SM. Circulating microRNAs (cmiRNAs) as novel potential biomarkers for hepatocellular carcinoma. Neoplasma. 2013; 60:135-142.

7. Huang Z, Huang D, Ni S, Peng Z, Sheng W, Du X: Plasma microRNAs are promising novel biomarkers for early detection of colorectal cancer. Int J Cancer. 2010; 127:118-126.

8. Liu R, Chen X, Du Y, Yao W, Shen L, Wang C, Hu Z, Zhuang R, Ning G, Zhang C, Yuan Y, Li Z, Zen K, et al. Serum microRNA expression profile as a biomarker in the diagnosis and prognosis of pancreatic cancer. Clin Chem. 2012; 58:610-618.

9. Pant S, Hilton H, Burczynski ME. The multifaceted exosome: biogenesis, role in normal and aberrant cellular function, and frontiers for pharmacological and biomarker opportunities. Biochem Pharmacol. 2012; 83:1484-1494.

10. Lasser C. Exosomal RNA as biomarkers and the therapeutic potential of exosome vectors. Expert Opin Biol Ther. 2012; 12 Suppl 1:S189-S197.

11. Overbye A, Skotland T, Koehler CJ, Thiede B, Seierstad T, Berge V, Sandvig K, Llorente A. Identification of prostate 
cancer biomarkers in urinary exosomes. Oncotarget. 2015; 6:30357-30376. http://doi.org/10.18632/oncotarget.4851.

12. Lima LG, Chammas R, Monteiro RQ, Moreira ME, Barcinski MA. Tumor-derived microvesicles modulate the establishment of metastatic melanoma in a phosphatidylserine dependent manner. Cancer Lett. 2009; 283:168-175.

13. Sohn W, Kim J, Kang SH, Yang SR, Cho JY, Cho HC, Shim SG, Paik YH. Serum exosomal microRNAs as novel biomarkers for hepatocellular carcinoma. Exp Mol Med. 2015; 47:e184.

14. Li Z, Ma YY, Wang J, Zeng XF, Li R, Kang W, Hao XK. Exosomal microRNA-141 is upregulated in the serum of prostate cancer patients. Onco Targets Ther. 2016; 9:139-148.

15. Taverna S, Giallombardo M, Gil-Bazo I, Carreca AP, Castiglia M, Chacartegui J, Araujo A, Alessandro R, Pauwels P, Peeters M, Rolfo C. Exosomes isolation and characterization in serum is feasible in non-small cell lung cancer patients: critical analysis of evidence and potential role in clinical practice. Oncotarget. 2016; 7:28748-60. http://doi.org/10.18632/oncotarget.7638.

16. Si H, Sun X, Chen Y, Cao Y, Chen S, Wang H, Hu C. Circulating microRNA-92a and microRNA-21 as novel minimally invasive biomarkers for primary breast cancer. J Cancer Res Clin Oncol. 2013; 139:223-229.

17. Nygren MK, Tekle $C$, Ingebrigtsen VA, Makela R, Krohn M, Aure MR, Nunes-Xavier CE, Perala M, Tramm T, Alsner $\mathrm{J}$, Overgaard $\mathrm{J}^{5}$, Nesland $\mathrm{JM}^{6}$, Borgen $\mathrm{E}$, et al. Identifying microRNAs regulating $\mathrm{B} 7-\mathrm{H} 3$ in breast cancer: the clinical impact of microRNA-29c. Br J Cancer. 2014; 110:2072-2080.

18. Chen J, Sun D, Chu H, Gong Z, Zhang C, Gong B, Li Y, Li N, Jiang L. Screening of differential microRNA expression in gastric signet ring cell carcinoma and gastric adenocarcinoma and target gene prediction. Oncol Rep. 2015; 33:2963-2971.

19. Murakami T, Kim T, Oi H, Nakamura H, Igarashi H, Matsushita M, Okamura J, Kozuka T. Detectability of hypervascular hepatocellular carcinoma by arterial phase images of MR and spiral CT. Acta Radiol. 1995; 36:372-376.

20. Jiang CF, Li DM, Shi ZM, Wang L, Liu MM, Ge X, Liu X, Qian YC, Wen YY, Zhen LL, Lin J, Liu LZ, Jiang BH. Estrogen regulates miRNA expression: implication of estrogen receptor and miR-124/AKT2 in tumor growth and angiogenesis. Oncotarget. 2016; 7:36940-36955. http://doi. org/10.18632/oncotarget.9230.

21. Wang Z, Xu L, Hu Y, Huang Y, Zhang Y, Zheng X, Wang S, Wang Y, Yu Y, Zhang M, Yuan K, Min W. miRNA let-7b modulates macrophage polarization and enhances tumorassociated macrophages to promote angiogenesis and mobility in prostate cancer. Sci Rep. 2016; 6:25602.

22. Zhi Q, Zhu J, Guo X, He S, Xue X, Zhou J, Hu B, Li H, Chen S, Zhao H, Kuang Y. Metastasis-related miR-185 is a potential prognostic biomarker for hepatocellular carcinoma in early stage. Biomed Pharmacother. 2013; 67:393-398.

23. Melo SA, Sugimoto H, O'Connell JT, Kato N, Villanueva A, Vidal A, Qiu L, Vitkin E, Perelman LT, Melo CA, Lucci
A, Ivan C, Calin GA, Kalluri R. Cancer exosomes perform cell-independent microRNA biogenesis and promote tumorigenesis. Cancer Cell. 2014; 26:707-721.

24. Kogure T, Lin WL, Yan IK, Braconi C, Patel T. Intercellular nanovesicle-mediated microRNA transfer: a mechanism of environmental modulation of hepatocellular cancer cell growth. Hepatology. 2011; 54:1237-1248.

25. Liu J, Sun H, Wang X, Yu Q, Li S, Yu X, Gong W. Increased exosomal microRNA-21 and microRNA-146a levels in the cervicovaginal lavage specimens of patients with cervical cancer. Int J Mol Sci. 2014; 15:758-773.

26. Liu WH, Ren LN, Wang X, Wang T, Zhang N, Gao Y, Luo H, Navarro-Alvarez N, Tang LJ. Combination of exosomes and circulating microRNAs may serve as a promising tumor marker complementary to alpha-fetoprotein for early-stage hepatocellular carcinoma diagnosis in rats. J Cancer Res Clin Oncol. 2015; 141:1767-1778.

27. Ying $X$, Wu Q, Wu X, Zhu Q, Wang X, Jiang L, Chen $\mathrm{X}$, Wang X. Epithelial ovarian cancer-secreted exosomal miR-222-3p induces polarization of tumor-associated macrophages. Oncotarget. 2016; 7:43076-43087. http://doi. org/10.18632/oncotarget.9246.

28. Sugimachi K, Matsumura T, Hirata H, Uchi R, Ueda M, Ueo H, Shinden Y, Iguchi T, Eguchi H, Shirabe K, Ochiya T, Maehara Y, Mimori K. Identification of a bona fide microRNA biomarker in serum exosomes that predicts hepatocellular carcinoma recurrence after liver transplantation. Br J Cancer. 2015; 112:532-538.

29. Soung YH, Nguyen T, Cao H, Lee J, Chung J. Emerging roles of exosomes in cancer invasion and metastasis. BMB Rep. 2016; 49:18-25.

30. Yu DD, Wu Y, Shen HY, Lv MM, Chen WX, Zhang XH, Zhong SL, Tang JH, Zhao JH. Exosomes in development, metastasis and drug resistance of breast cancer. Cancer Sci. 2015; 106:959-964.

31. Skog J, Wurdinger T, van Rijn S, Meijer DH, Gainche L, Sena-Esteves M, Curry WJ, Carter BS, Krichevsky AM, Breakefield XO. Glioblastoma microvesicles transport RNA and proteins that promote tumour growth and provide diagnostic biomarkers. Nat Cell Biol. 2008; 10:1470-1476.

32. Liu C, Yu S, Zinn K, Wang J, Zhang L, Jia Y, Kappes JC, Barnes S, Kimberly RP, Grizzle WE, Zhang HG. Murine mammary carcinoma exosomes promote tumor growth by suppression of NK cell function. J Immunol. 2006; 176:1375-1385.

33. Han Y, Wu Z, Wu T, Huang Y, Cheng Z, Li X, Sun T, Xie X, Zhou Y, Du Z. Tumor-suppressive function of long noncoding RNA MALAT1 in glioma cells by downregulation of MMP2 and inactivation of ERK/MAPK signaling. Cell Death Dis. 2016; 7:e2123.

34. Carlo-Stella C, Locatelli SL, Giacomini A, Cleris L, Saba E, Righi M, Guidetti A, Gianni AM. Sorafenib inhibits lymphoma xenografts by targeting MAPK/ERK and AKT pathways in tumor and vascular cells. PLoS One. 2013; 8:e61603.

35. Qu JL, Qu XJ, Zhao MF, Teng YE, Zhang Y, Hou KZ, Jiang YH, Yang XH, Liu YP. Gastric cancer exosomes promote 
tumour cell proliferation through PI3K/Akt and MAPK/ ERK activation. Dig Liver Dis. 2009; 41:875-880.

36. Ghayad SE, Cohen PA. Inhibitors of the PI3K/Akt $/ \mathrm{mTOR}$ pathway: new hope for breast cancer patients. Recent Pat Anticancer Drug Discov. 2010; 5:29-57.

37. Wang W, Zou L, Zhou D, Zhou Z, Tang F, Xu Z, Liu X. Overexpression of ubiquitin carboxyl terminal hydrolase-L1 enhances multidrug resistance and invasion/metastasis in breast cancer by activating the MAPK/Erk signaling pathway. Mol Carcinog. 2016; 55:1329-42.
38. Setia S, Nehru B, Sanyal SN. Upregulation of MAPK/Erk and PI3K/Akt pathways in ulcerative colitis-associated colon cancer. Biomed Pharmacother. 2014; 68:1023-1029.

39. Valadi H, Ekstrom K, Bossios A, Sjostrand M, Lee JJ, Lotvall JO. Exosome-mediated transfer of mRNAs and microRNAs is a novel mechanism of genetic exchange between cells. Nat Cell Biol. 2007; 9:654-659. 